\title{
Inflammasomes in Pediatric Autoinflammatory Diseases with Recurrent Fever
}

\author{
Umut Gazi' ${ }^{1}$, Nerin N. Bahçeliler² (1) \\ 'Department of Medical Microbiology and Clinical Microbiology, Near East University School of Medicine, Nicosia, Cyprus \\ ${ }^{2}$ Department of Pediatrics, Near East University School of Medicine, Nicosia, Cyprus
}

ORCID IDs of the authors: U.G. 0000-000I-9945-478X; N.N.B. 0000-000I-842I-3625.

Cite this article as: Gazi U, Bahçeliler NN. Inflammasomes in Pediatric Autoinflammatory Diseases with Recurrent Fever. Cyprus J Med Sci 2019; 4(2): I3I-5.

Recurrent fever is common in young, especially preschool, children, and is associated with repeated episodes of fever that, in some cases, may last for weeks. Most often, these episodes are due to repeated infections; however, when there is a periodic recurrence, they can also be because of conditions such as autoinflammatory diseases (AIDs). Since their discovery nearly two decades ago, AIDs received a growing interest in research studies that not only increased our understanding of the AID pathologies, but also enriched the literature on innate immune responses, including inflammasome-mediated pathways. Inflammasomes are protein complexes formed in response to the activation of a group of intracellular pathogen-recognition receptors by a variety of inducers originating from the infection or cellular stress. They are involved in the generation of caspase-l, which is required for the generation of active interleukin (IL)-I $\beta$ and IL-18, and were also shown to induce the generation of a wide variety of other cytokines, including tumor necrosis factor (TNF)- $\alpha$, IL-6, and interferons (IFNs). This review aims not only to raise awareness about pediatric AIDs with periodic fever, but also to present the current literature about the molecular basis of inflammasome-mediated pathways involved in the disease pathogenesis. Future studies that would further enlighten the relationship between inflammasome-mediated pathways and AIDs would contribute to the development of more effective treatment strategies aiming to improve the patient life quality and help to avoid long-term complications.

Keywords: Autoinflammatory diseases, inflammasomes, periodic fever

\section{INTRODUCTION}

The term autoinflammatory disease was first used in a study by Galon in 1999, which also identified the gene responsible for tumor necrosis factor (TNF) receptor-associated periodic syndrome (TRAPS). Autoinflammatory diseases (AIDs) were initially distinguished from autoimmune diseases by their characteristic feature of apparently unprovoked inflammation episodes without any auto-reactive T lymphocyte or auto-antibody induction (I). However, today it became apparent that both innate and adaptive immune responses can be activated during AIDs, and therefore, a new continuum model was suggested by McDermott and McGonagle in which immunological diseases are regarded as a continuum, with "pure monogenic autoinflammatory diseases" and "pure monogenic autoimmune diseases" being located at opposite ends (2).

Nevertheless, AIDs are considered to be mainly innately immune driven and are associated with neutrophil, macrophage, or monocyte-mediated inflammation with dysregulated cytokine production. They are also characterized by systemic inflammation, influencing tissues such as the skin, joints, conjunctiva, and serosal tissues (I). Over the last 20 years, the studies conducted on AIDs not only helped to improve our understanding of the AID pathogenesis, but also of the innate immune mechanisms, namely inflammasomes, which have also been linked to autoinflammatory, as well as autoimmune diseases.

\section{Inflammasomes}

Innate immune responses are triggered as a result of the interaction between pattern recognition receptors (PRRs) and pathogen-associated molecular patterns (PAMPs), or damage-associated molecular patterns (DAMPs). Depending on their localization, PRRs can be divided into two broad families: (I) transmembrane receptors (e.g., Toll-like receptors [TLRs]) that are found in the plasma membrane and in endosomes, and (2) PRRs, which are expressed in intracellular compartments (e.g, nucleotide-binding domain and leucine-rich repeat-containing proteins [NLRP]). 
In response to a variety of stimuli originating from infection or cellular stress, while some intracellular PRRs, activate nuclear

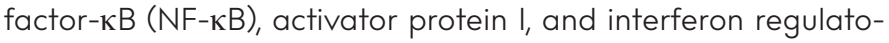
ry factors, some stimulate the assembly of protein complexes called inflammasomes. A typical inflammasome is composed of a sensor protein, which is an intracellular PRR; apoptosis-associated speck-like (ASC) protein that acts as an adaptor protein and possesses a caspase-recruitment domain (CARD); and procaspase-l. Some inflammasomes were also reported to include chaperone proteins (e.g., heat shock protein), which are thought to play an important role in stabilizing the protein-protein interactions (3). Up until today, there have been at least seven types of inflammasomes identified, and each is named according to the intracellular PRR associated: NLRPI, NLRP3, NLRP6, NLRPI2, NLR Family CARD Domain Containing 4 (NLRC4), retinoic acid-inducible gene (RIG)-I, and absent in melanoma (AIM)-2. Among those, the NLRP3-inflammasome is regarded as the most important one as it is responsive to a wide range of PAMPs and DAMPs (4).

Upon detection of an activating signal, inflammasome sensors bind pro-caspase-I either directly via CARD or indirectly via the pyrin domain (PYD), which interacts with ASC that has both the PYD and CARD domains. Pro-caspase then undergoes oligomerization in inflammasomes, as well as the autoproteolytic cleavage that yields caspase-I (4). Active caspase-I is a cysteine-dependent protease that is involved in the generation of interleukin (IL)-I $\beta$ and IL-I8 from pro-IL-I $\beta$ and pro-IL-I8, respectively. While both cytokines are involved in inflammatory diseases, infection, and cancer, IL-I $\beta$ is regarded as one of the major mediators of fever in humans and is regarded as a "prototypic alarm cytokine" because of its ability to induce the NF-кB activation and inflammatory cytokine release. In addition to the IL-I family member cytokines, inflammasomes were also associated with the induction of other wide variety of cytokines such as TNF- $\alpha$, IL-6 IL-II, IL-I7A, interferon (IFN)- $\alpha$, and IFN- $\beta$ (5).

Caspase-I activation can also lead to pyroptosis, which is an inflammatory form of cell death associated with the release of proinflammatory cellular contents due to rupture of the plasma membrane. Its activation results in the pore formation on the plasma membrane, which disrupts the cellular ionic gradient causing increased osmotic pressure, swelling, and consequently, lysis. For this reason, caspase-l is suggested to help host immune defense by both mediating the cytokine release and destruction of infected or damaged host cells (6).

\section{Inflammasomes and Pediadtric AIDs with Fever}

Recurrent fever is a common clinical presentation observed in preschool children, and is characterized by repeated episodes of fever that last for days or weeks with symptom-free intervals for weeks or months. While it is mostly caused by repeated infections, in cases of continued recurrence, it is suspected to be due to other conditions such as primary immunodeficiencies, anatomic and metabolic abnormalities, malignancies, and AIDs (5).

Fever is common among AIDs; however, some cases are associated with other prominent features, including pyogenic and granulomatous lesions, and are classified accordingly. On the other hand, AIDs associated with repeated fever episodes are classified differently and include monogenic forms of autoinflammation, as well as genetically complex autoinflammatory diseases such as the periodic fever, aphthous stomatitis, pharyngitis, and cervical adenitis (PFAPA) syndrome (7).

\section{Hereditary Periodic Fever Syndromes}

\section{Familial mediterranean fever (FMF)}

Familial mediterranean fever is the most common monogenic inflammatory disease and is characterized by the episodes of fever, polyserositis abdominanal pain, and long-term complication of amyloidosis. The affected gene, known as the Mediterranean fever gene (MEFV), encodes the pyrin protein, which is involved in the inflammasome formation, regulation of apoptosis, inflammation, and IL-I $\beta$ processing (8). The protein was also suggested to facilitate the autophagic degradation of inflammasome components including NLRPI, NLRP3, and pro-caspase I (9).

Up until today, there had been over 310 different mutations associated with FMF, most of which were positioned in the C-terminal B30.2 (PYR) domain involved in the protein-protein interactions (I0). The most common FMF-associated mutations are M694V, M694I, V726A, M680I, and El48Q, which account for $70 \%$ of cases in the Mediterranean population (II). Among those, the M694V and EI48Q mutations are responsible for severe and milder forms of the disease, respectively (8). FMF is known to primarily affect the Mediterranean populations, and our recent study demonstrated a high MEFV gene mutation carrier rate of $12.5 \%$ in the Turkish Cypriot population (12).

Albeit FMF was initially associated with the loss-of-function autosomal recessive mutations, previous studies reported that $30 \%$ of the FMF patients possessed only a single demonstrable mutation, and mice inserted with FMF-associated human B30.2 domains displayed phenotypes similar to those observed in patients with the FMF phenotype, while deletion of mouse pyrin did not result in any inflammatory phenotype $(13,14)$. Additionally, monocytes isolated from patients with FMF were demonstrated to release higher LPS-induced levels of IL-I $\beta$ than those from healthy donors (I5).

While these studies suggest that the gain-of-function mutations are responsible for the disease, Jamilloux et al. (16) showed that the disease is associated with hypermorphic mutations that result in reduction of the activation threshold of the pyrin inflammasome. Moreover, the B30.2 domain mutations were shown to decrease the activation threshold by inhibiting pyrin phosphorylation by pyrin kinases, and thereby reducing the 14-3-3 binding, which is involved in blocking of the inflammasome activity (17). On the other hand, the ASC-dependent NLRP3-independent inflammasomes were also suggested to facilitate the FMF development in mouse models (14).

\section{Cryopyrin-associated periodic syndromes (CAPS)}

Cryopyrin-associated periodic syndromes is a broad term used to cover a group of autosomal-dominant inherited diseases, including familial cold autoinflammatory syndrome(FCAS), Muckle-Wells syndrome (MWS), and neonatal onset multisystem inflammatory disease (NOMID), among which FCAS and NOMID are the least and most severe forms, respectively. The symptoms include fever, rash, headache, and conjunctivitis. Unique fea- 
tures include cold-induced fever in FCAS; hearing loss in MWS and NOMID; overgrowth of knees; and symptoms associated with the central nervous system inflammation (CNS) of patients with NOMID. If left untreated, amyloidosis leading to end-stage renal disease can also be observed in patients with MWS (3).

While they were initially thought to be caused by distinct mechanisms, later studies reported that CAPS were all associated with the NLRP3 inflammasome (18). Up to date, over 175 different NLRP3 gene sequence variants and over 90 heterozygous NLRP3 mutations were associated with CAPS disease (19). It is a rare disease (prevalence of approximately I-3 per I million), predominantly affecting the Northern European population (20).

An in vitro study reported increased levels of lipopolysaccharide (LPS)-induced IL-I $\beta$ and IL-I8 production in the caspase-I-dependent manner in CAPS patients, when compared with that from control subjects (2I). Additionally, in contrast to monocytes from control patients, the FCAS patient monocytes were able to release $\mathrm{IL}-\mathrm{I} \beta$ following incubation at $32^{\circ} \mathrm{C}$ which is the skin temperature of subjects exposed to cold (22). In the same study, the generation of several cytokines was IL-I $\beta$ dependent, as reported by reduction in the levels following treatment with an IL-I receptor antagonist (22). In another study, a fast IL-I $\beta$ production by CAPS patients was associated with an altered redox state, as demonstrated by enhanced reactive oxygen species (ROS) and antioxidant levels in the CAPS monocytes that were left unstimulated (23). Moreover, macrophages from mice expressing CAPS mutations also displayed enhanced levels of the IL-I $\beta$ and IL-I8 production in response to TLR ligands in the absence of ATP. This was thought to be due to a reduced inflammasome activation threshold, which could be as a result of conformation changes introduced by the associated mutation (24).

\section{Mevalonate kinase deficiency (MKD)}

Mevalonate kinase deficiency is a rare disease with two phenotypes that include hyperimmunoglobulinemia $D$ syndrome (HIDS), which is less severe but more common; and mevalonic aciduria (MVA), which is a more severe but a less common form (25). MKD presents with recurrent inflammatory attacks, with a sudden onset of high fever that may last up to 6 days, which is usually accompanied by other features including lymphadenopathy and gastrointestinal, mucocutaneous, musculoskeletal, and neurological symptoms (7). In a severe MKD form, children may also suffer from growth retardation, congenital defects, psychomotor retardation, progressive cerebellar ataxia, and hypotonia (25).

It is inherited in an autosomal recessive manner and is caused by a mutation of the mevalonate kinase (MVK) gene. While the MVK activity is usually below the detection levels in cultured fibroblast cells isolated from MVA, it is approximately $1 \%-7 \%$ of the control value in fibroblasts and leukocytes from patients with HIDS (26).

The MVK protein is important for the mevalonate pathway of cholesterol and isoprene biosynthesis, and its mutation in MKD leads to reduced mevalonate-derived intermediates, including geranylgeranyl pyrophosphate (GGPP). GGPP is the geranylgeranylation substrate required for the membrane targeting of ras homolog gene family member $A$ (RhoA), which is involved in the regulation of the pyrin inflammasome activity via kinases that block pyrin effects by phosphorylation (7, 17,27). Accordingly, pyrin inflammasomes were shown to be activated in response to Rho inactivation, and the inhibition of mevalonate pathway was reported to elevate the NALP3 expression, which suggests its involvement in $\operatorname{MKD}(7,27,28)$.

While its epidemiology is still unclear, and its incidence rate seems to vary depending on the region, the Netherlands is reported to have the highest prevalence with an incident rate of l:200,000 (29). MKD is a very rare disease, and there are approximately 300 MKD patients known today. However, this is more likely an underestimated number due to lack of genetic screening programs for MKD, as well as undocumented and undiagnosed patients that stay unreported in literature (25).

\section{Tumor necrosis factor receptor-associated periodic fever syn- drome (TRAPS)}

TRAPS, which was initially known as Hibernian fever, was first described in 1982 in a boy of Irish-Scottish origin (30). It is regarded as the most common autosomal-dominant AID with symptoms including fever, abdominal, chest and testicular pain, conjunctivitis, arthralgia, and myalgia, which may last up to 3 weeks or even longer. In addition to those, some patients may also suffer from periorbital edema and painful migratory erythematous rash (20). It is caused by the missense mutation of the TNF receptor (TNFR)-I extracellular domain that influences the folding and trafficking of the protein (3I).

Even though the gene responsible for TRAPS is not a part of inflammasomes, inflammasome-mediated pathways are still thought to be involved in the pathogenesis since ROS, which acts as a driver in the inflammation responsible for TRAPS, is known to contribute to the NLRP3 inflammasome activation $(32,33)$. In another study, mutant TNRF-I was demonstrated to be trapped within the cell and increase the LPS-induced MAPK activation, as well as the proinflammatory cytokine secretion levels (3I). Moreover, TNF- $\alpha$ was also reported to induce the inflammasome activation in the absence of any bacterial infection and was shown to act as an important transcriptional regulator of the NLRP3 inflammasome components $(34,35)$. On the other hand, while literature on the prevalence of TRAPS is still scarce, its incidence rate among the pediatric population in Germany was estimated at approximately 5.6 in $10^{7}$ persons (36).

\section{Familial cold autoinflammatory syndrome 2 (FCAS 2)}

Familial cold autoinflammatory syndrome 2 is a very rare genetic disease associated with NLRI2 mutations that is transmitted with autosomal-dominant inheritance. Current literature on the effect of this mutation on the immune response is not clear: Jeru et al. (37) reported a deleterious effect on the NF-KB signaling; however, Borghini et al. (38) did not observe such an inhibitory effect. On the other hand, its possible effect on the noncanonical $\mathrm{NF}-\kappa \mathrm{B} / \mathrm{pl00}$ processing pathway is still unclear (38). The clinical symptoms resemble those reported for FCAS and include fever that may last up to 10 days, together with headache, joint symptoms, and skin rash triggered by the exposure to cold. In some cases, urticaria, abdominal pain and lymphadenopathy, and hearing loss were also observed (39). 


\section{PFAPA}

PFAPA, which is named according to its symptoms that include fever flares associated with pharyngitis, adenitis, and/or aphthous stomatitis in the absence of any infection, is the most common pediatric AID in regions without a high FMF prevalence and is known as the most common cause of recurrent fever among the pediatric population in Europe $(40,41)$. It is considered as a self-limited condition as it generally fades away before adulthood (7).

Today, even though the cause of the disease is not clear, it is thought to be associated with dysregulated innate immunity as the patients exhibit increased serum levels of proinflammatory cytokines, neutrophilia, and are responsive to corticosteroids and IL-I blockade (2). Accordingly, in our study, tonsil samples isolated from patients with PFAPA displayed similar antimicrobial peptide expression levels when compared with those isolated from the group of patients with A beta-hemolytic streptococcal recurrent tonsillitis (42). Tonsils seem to play a crucial role in PFAPA since tonsillectomy was shown to be an effective treatment strategy for the PFAPA disease, and tonsillar microbiome was shown to differ between the control subjects and patients with PFAPA (43-45).

In contrast to most of the periodic fever syndromes, which were shown to be hereditary monogenic disorders, the genetic basis for PFAPA is not yet clear. Recent studies that examined siblings and related mothers suggested a familial susceptibility and demonstrated an association between PFAPA and some candidate genes. However, lack of a clear monogenic trait suggests heterogenous, polygenic, or complex inheritance of PFAPA syndrome (46).

Among those are included inflammasome-related genes, mostIy NLRP3 and MEFV variants, which implies inflammasome-mediated pathways in the PFAPA development (47). In support of its role, PFAPA was also reported to be associated with the CARD8 mutation that disrupts its binding to the NLRP3 inflammasome and thereby reducing its negative regulatory activity $(40,48)$. This correlates with previous reports showing increased IL-I $\beta$ expression during PFAPA febrile attacks $(49,50)$.

\section{CONCLUSION}

Since the discovery of AID, the literature on the disease pathogenesis and innate immunity including inflammasome-mediated pathways have proceeded hand in hand, such that due to its important role in inflammation, IL-I blocking strategy is being used to treat AID with periodic fever (5). Today clinical application of this strategy involves using medications that either target the IL-I molecule directly (e.g., canakinumab, rilonacept, gevokizumab) or indirectly via antagonistic effects on the IL-I receptor (e.g., anakinra) (5). Nevertheless, as the inflammasome activity is also needed for host response to microbial pathogens, a greater understanding of the balance between beneficial and detrimental inflammasome activation would contribute to the discovery of new and better inflammasome-focused treatment strategies.

Peer-review: Externally peer-reviewed.

Author contributions: Concept - U.G., N.N.B.; Design - U.G.; Supervision - N.N.B.; Analysis and/or Interpretation - U.G., N.N.B.; Literature Search U.G., N.N.B.; Writing - U.G.; Critical Reviews - N.N.B.
Conflict of Interest: The authors have no conflicts of interest to declare.

Financial Disclosure: The authors declared that this study has received no financial support.

\section{REFERENCES}

I. Ciccarelli F, Martinis M, Ginaldi L. An Update on Autoinflammatory Diseases. Curr Med Chem 2013; 21: 26I-9. [CrossRef]

2. Wekell P, Karlsson A, Berg S, Fasth A. Review of autoinflammatory diseases, with a special focus on periodic fever, aphthous stomatitis, pharyngitis and cervical adenitis syndrome. Acta Paediatr 2016; 105: ||40-51. [CrossRef]

3. Hoffman HM, Broderick L. The role of the inflammasome in patients with autoinflammatory diseases. J Allergy Clin Immunol 2016; 138: 3-14. [CrossRef]

4. de Zoete MR, Palm NW, Zhu S, Flavell RA. Inflammasomes. Cold Spring Harb Perspect Biol 2014; 6: a016287. [CrossRef]

5. Moll M, Kuemmerle-Deschner JB. Inflammasome and cytokine blocking strategies in autoinflammatory disorders. Clin Immunol 20I3; 147: 242-75. [CrossRef]

6. Bergsbaken T, Fink SL, Cookson BT. Pyroptosis: Host cell death and inflammation. Nat Rev Microbiol 2009; 7: 99-109. [CrossRef]

7. Sag E, Bilginer Y, Ozen S. Autoinflammatory Diseases with Periodic Fevers. Curr Rheumatol Rep 2017; 19: 41. [CrossRef]

8. Manukyan G, Aminov R. Update on pyrin functions and mechanisms of familial mediterranean fever. Front Microbiol 2016; 7: 456. [CrossRef]

9. Kimura T, Jain A, Choi SW, Mandell MA, Schroder K, Johansen T, et al. TRIM-mediated precision autophagy targets cytoplasmic regulators of innate immunity. J Cell Biol 2015; 210: 973-89. [CrossRef]

10. Van Gorp H, Saavedra PH V, de Vasconcelos NM, Van Opdenbosch N, Vande Walle L, Matusiak M, et al. Familial Mediterranean fever mutations lift the obligatory requirement for microtubules in Pyrin inflammasome activation. Proc Natl Acad Sci U S A 20I6; II3: 14384-9. [CrossRef]

II. Fujikura K. Global epidemiology of Familial Mediterranean fever mutations using population exome sequences. Mol Genet Genomic Med 20I5; 3: 272-82. [CrossRef]

12. Galip N, Dalkan C, Terali A, Çobanoğlu N, Ülgenalp A, Bahçeciler N, et al. Prevalence of Mediterranean FeVer Gene Mutations in Turkish Cypriot Population. Arch Rheumatol 2017; 32: 10-4. [CrossRef]

13. Booty MG, Jae JC, Masters SL, Remmers EF, Barham B, Le JM, et al. Familial Mediterranean fever with a single MEFV mutation: Where is the second hit? Arthritis Rheum 2009; 60: 1851-61. [CrossRef]

14. Chae JJ, Cho YH, Lee GS, Cheng J, Liu PP, Feigenbaum L, et al. Gainof-Function Pyrin Mutations Induce NLRP3 Protein-Independent Interleukin-I $\beta$ Activation and Severe Autoinflammation in Mice. Immunity 20II; 34: 755-68. [CrossRef]

15. Omenetti A, Carta S, Delfino L, Martini A, Gattorno M, Rubartelli A. Increased NLRP3-dependent interleukin I $\beta$ secretion in patients with familial Mediterranean fever: Correlation with MEFV genotype. Ann Rheum Dis 2014; 73: 462-9. [CrossRef]

16. Jamilloux Y, Lefeuvre L, Magnotti F, Martin A, Benezech S, Allatif O, et al. Familial Mediterranean fever mutations are hypermorphic mutations that specifically decrease the activation threshold of the Pyrin inflammasome. Rheumatology (Oxford) 2018; 57: 100-II. [CrossRef]

17. Park YH, Wood G, Kastner DL, Chae JJ. Pyrin inflammasome activation and RhoA signaling in the autoinflammatory diseases FMF and HIDS. Nat Immunol 2016; 17: 914-21. [CrossRef]

18. Gurung P, Kanneganti TD. Autoinflammatory Skin Disorders: The Inflammasome in Focus. Trends Mol Med 2016; 22: 545-64. [CrossRef]

19. Kuemmerle-Deschner JB. Caps-pathogenesis, presentation and treatment of an autoinflammatory disease. Semin Immunopathol 2015; 37: 377-85. [CrossRef]

20. Ostring GT, Singh-Grewal D. Periodic fevers and autoinflammatory syndromes in childhood. J Paediatr Child Health 2016; 52: 865-7l. [CrossRef] 
21. Stack JH, Beaumont K, Larsen PD, Straley KS, Henkel GW, Randle JC, et al. IL-Converting Enzyme/Caspase-I Inhibitor VX-765 Blocks the Hypersensitive Response to an Inflammatory Stimulus in Monocytes from Familial Cold Autoinflammatory Syndrome Patients. J Immunol 2005; 175: 2630-4. [CrossRef]

22. Rosengren S, Mueller JL, Anderson JP, Niehaus BL, Misaghi A, Anderson $\mathrm{S}$, et al. Monocytes from familial cold autoinflammatory syndrome patients are activated by mild hypothermia. J Allergy Clin Immunol 2007; 119: 991-6. [CrossRef]

23. Tassi S, Carta S, Delfino L, Caorsi R, Martini A, Gattorno M, et al. Altered redox state of monocytes from cryopyrin-associated periodic syndromes causes accelerated IL-I secretion. Proc Natl Acad Sci 2010; 107: 9789-94. [CrossRef]

24. Meng G, Zhang F, Fuss I, Kitani A, Strober W. A Mutation in the Nlrp3 Gene Causing Inflammasome Hyperactivation Potentiates Thl7 Cell-Dominant Immune Responses. Immunity 2009; 30: 860-74. [CrossRef]

25. Zhang S. Natural history of mevalonate kinase deficiency: A literature review. Pediatr Rheumatol Online J 20I6; 14: 30. [CrossRef]

26. Houten SM, Schneiders MS, Wanders RJA, Waterham HR. Regulation of isoprenoid/cholesterol biosynthesis in cells from mevalonate kinase-deficient patients. J Biol Chem 2003; 278: 5736-43. [CrossRef]

27. Pontillo A, Paoluzzi E, Crovella S. The inhibition of mevalonate pathway induces upregulation of NALP3 expression: New insight in the pathogenesis of mevalonate kinase deficiency. Eur J Hum Genet 20I0; 18: 844-7. [CrossRef]

28. Xu H, Yang J, Gao W, Li L, Li P, Zhang L, et al. Innate immune sensing of bacterial modifications of Rho GTPases by the Pyrin inflammasome. Nature 20I4; 5I3: 237-4I. [CrossRef]

29. Favier LA, Schulert GS. Mevalonate kinase deficiency: Current perspectives. Appl Clin Genet 2016; 9: 101-10. [CrossRef]

30. Ogoina D. Fever, fever patterns and diseases called "fever" - A review. J Infect Public Health 20II; 4: 108-24. [CrossRef]

31. Simon A, Park H, Maddipati R, Lobito AA, Bulua AC, Jackson AJ, et al. Concerted action of wild-type and mutant TNF receptors enhances inflammation in TNF receptor I-associated periodic fever syndrome. Proc Natl Acad Sci 2010; 107: 980I-6. [CrossRef]

32. Zhou R, Tardivel A, Thorens B, Choi I, Tschopp J. Thioredoxin-interacting protein links oxidative stress to inflammasome activation. Nat Immunol 2010; II: I36-40. [CrossRef]

33. Bulva AC, Pelletier M, Myerowitz-Vanderhoek S, Kastner D, Siegel RM. Mitochondrial reactive oxygen species promote production of proinflammatory cytokines in the TNFRI-associated periodic fever syndrome. Inflamm Res 20II; 60: S79. [CrossRef]

35. McGeough MD, Wree A, Inzaugarat ME, Haimovich A, Johnson CD, $\mathrm{Pe}-\mathrm{a} \mathrm{CA}$, et al. TNF regulates transcription of NLRP3 inflammasome components and inflammatory molecules in cryopyrinopathies. J Clin Invest 2017; 127: 4488-97. [CrossRef]

36. de Jesus AA, Goldbach-Mansky R. Monogenic autoinflammatory diseases: Concept and clinical manifestations. Clin Immunol 2013; 147: 155-74. [CrossRef]
37. Jeru I, Duquesnoy P, Fernandes-Alnemri T, Cochet E, Yu JW, LackmyPort-Lis M, et al. Mutations in NALPI2 cause hereditary periodic fever syndromes. Proc Natl Acad Sci U S A 2008; 105: 1614-9. [CrossRef]

38. Borghini S, Tassi S, Chiesa S, Caroli F, Carta S, Caorsi R, et al. Clinical presentation and pathogenesis of cold-induced autoinflammatory disease in a family with recurrence of an NLRPI2 mutation. Arthritis Rheum 20II; 63: 830-9. [CrossRef]

39. Ozen S, Bilginer Y. A clinical guide to autoinflammatory diseases: Familial Mediterranean fever and next-of-kin. Nat Rev Rheumatol 2014; 10: 135-47. [CrossRef]

40. Cheung MS, Theodoropoulou K, Lugrin J, Martinon F, Busso N, Hofer M. Periodic Fever with Aphthous Stomatitis, Pharyngitis, and Cervical Adenitis Syndrome Is Associated with a CARD8 Variant Unable To Bind the NLRP3 Inflammasome. J Immunol 2017; 198: 2063-9. [CrossRef]

4l. Toplak N, Frenkel J, Ozen S, Lachmann HJ, Woo P, Koné-Paut l, et al An international registry on autoinflammatory diseases: The Eurofever experience. Ann Rheum Dis 2012; 71: II77-82. [CrossRef]

42. Gazi U, Agada ME, Ozkayalar H, Dalkan C, Sanlidag B, Safak MA, et al. Tonsillar antimicrobial peptide (AMP) expression profiles of periodic fever, aphthous stomatitis, pharyngitis, cervical adenitis (PFAPA) patients. Int J Pediatr Otorhinolaryngol 2018; II0: 100-4. [CrossRef]

43. Tasher D, Somekh E, Dalal I. PFAPA syndrome: new clinical aspects disclosed. Arch Dis Child 2006; 91: 981-4. [CrossRef]

44. Lantto U, Koivunen P, Tapiainen T, Glumoff V, Hirvikoski P, Uhari M, et al. Microbes of the tonsils in PFAPA (Periodic Fever, Aphtous stomatitis, Pharyngitis and Adenitis) syndrome - A possible trigger of febrile episodes. APMIS 2015; 123: 523-9. [CrossRef]

45. Tejesvi M V., Uhari M, Tapiainen T, Pirttilä AM, Suokas M, Lantto $U$, et al. Tonsillar microbiota in children with PFAPA (periodic fever, aphthous stomatitis, pharyngitis, and adenitis) syndrome. Eur J Clin Microbiol Infect Dis 2016; 35: 963-70. [CrossRef]

46. Kraszewska-Głomba B, Matkowska-Kocjan A, Szenborn L. The Pathogenesis of Periodic Fever, Aphthous Stomatitis, Pharyngitis, and Cervical Adenitis Syndrome: A Review of Current Research. Mediators Inflamm 2015; 2015: 563876. [CrossRef]

47. Vanoni F, Theodoropoulou K, Hofer M. PFAPA syndrome: A review on treatment and outcome. Pediatr Rheumatol Online J 2016; 14: 38. [CrossRef]

48. Ito $S$, Hara $Y$, Kubota T. CARD8 is a negative regulator for NLRP3 inflammasome, but mutant NLRP3 in cryopyrin-associated periodic syndromes escapes the restriction. Arthritis Res Ther 2014; 16: R52. [CrossRef]

49. Kolly L, Busso N, Von Scheven-Gete A, Bagnoud N, Moix I, Holzinger $D$, et al. Periodic fever, aphthous stomatitis, pharyngitis, cervical adenitis syndrome is linked to dysregulated monocyte IL-I $\beta$ production. J Allergy Clin Immunol 20I3; 131: 1635-43. [CrossRef]

50. Stojanov S, Lapidus S, Chitkara P, Feder H, Salazar JC, Fleisher TA, et al. Periodic fever, aphthous stomatitis, pharyngitis, and adenitis (PFAPA) is a disorder of innate immunity and Thl activation responsive to IL-I blockade. Proc Natl Acad Sci U S A 20ll; 108: 714853. [CrossRef] 